\title{
Dramatic enhancement in energy harvesting for a narrow range of dimensions in piezoelectric nanostructures
}

\author{
M. S. Majdoub, ${ }^{1}$ P. Sharma, ${ }^{1,2, *}$ and T. Çağin ${ }^{3}$ \\ ${ }^{1}$ Department of Mechanical Engineering, University of Houston, Houston, Texas 77204, USA \\ ${ }^{2}$ Department of Physics, University of Houston, Houston, Texas 77204, USA \\ ${ }^{3}$ Department of Chemical Engineering, Texas A\&M University, College Station, Texas 77845, USA
}

(Received 2 July 2008; published 25 September 2008)

\begin{abstract}
Recent work suggests that flexoelectricity causes significant enhancement of electromechanical coupling of nonuniformly strained piezoelectric and nonpiezoelectric nanostructures below a material-dependent length scale. In the present work, employing an atomistically informed dynamical continuum model that accounts for flexoelectricity, we argue that in a narrow range of geometric dimensions, piezoelectric nanostructures can dramatically enhance energy harvesting. Specifically, in the case of lead zirconate titanate (PZT) material employed in the form of cantilever beams, our results indicate that the total harvested power peak value can increase by $100 \%$ around $21 \mathrm{~nm}$ beam thickness (under short circuit conditions) and nearly a 200\% increase may be achieved for specifically tailored cross-section shapes. The key (hereto undiscovered) insight is that the striking enhancement in energy harvesting is predicted to rapidly diminish (compared to bulk) both below and above a certain nanoscale structural length thus providing a rather stringent condition for the experimentalists.
\end{abstract}

DOI: 10.1103/PhysRevB.78.121407

PACS number(s): $61.46 . \mathrm{Km}, 77.90 .+\mathrm{k}, 84.60 .-\mathrm{h}, 85.85 .+\mathrm{j}$

Piezoelectricity, the development of electrical polarization upon uniform mechanical stimulus and thus the conversion of mechanical to electrical energy, has received intense attention due to numerous applications ranging from simple consumer products such as cell phones to complex technological advancements: atomic force microscopy, sensors and actuators [e.g., microelectromechanical systems (MEMs)], sonars, and artificial muscles among many others. In crystalline dielectrics, piezoelectricity can only occur in noncentrosymmetric systems. Under applied uniform strains, the absence of a center of symmetry about the positive and negative ions of the crystal structure induces electrical dipole moments and hence a polarization. The converse effect involves mechanical deformation upon actuation with an applied electric field.

Not surprisingly, these classes of materials have found applications in energy conversion as well. In line with recent advances in electronics, there is an increasing interest in scavenging and converting environmental energy into exploitable electric energy. Applications of these notions are anticipated in a wide variety of consumer, military, and specialized applications. For example, many researchers have recently investigated the use of piezoelectric transducers to harness human walking energy from shoe inserts ${ }^{1,2}$ and backpacks. ${ }^{3}$ Such energy can be used to power wearable electronics and charge batteries that can ideally be selfpowered with extended life span. The reader may refer to the reviews by Sodano et al., ${ }^{4,5}$ Beeby et al., ${ }^{6}$ and Priya ${ }^{7}$ on recent achievements in power harvesting by piezoelectric devices. Roundy et $a l{ }^{8}$ also provided a useful comparison and a discussion of the different energy scavenging devices from ambient sources and give particular attention to piezoelectric cantilever designs with new microscale geometries. As the dimension of the different functional devices is reduced down to nanoscale, the need for efficient piezoelectric energy harvesting nanodevices is becoming more and more critical. Recently, a controlled experimental method for the study of direct piezoelectric effect in perovskites such as $\mathrm{ZnO}$ (Refs.
9-11) and $\mathrm{BaTiO}_{3}$ (Ref. 12) nanostructures has been successfully demonstrated for efficient energy harvesting applications. Wang et al..$^{9-11}$ were able to bend the tips of aligned arrays of piezoelectric cantilever zinc oxide nanowires with a conductive atomic force microscope in contact mode and through ultrasonic waves. When used as a piezoelectric power generator the efficiency of the cantilever nanowire device is estimated to reach $30 \%$. In the present work, we argue that a dramatic enhancement is achievable in the harvested power of piezoelectric cantilever nanobeams through exploitation of size effects at the nanoscale under inhomogeneous strain, provided their thicknesses fall within a narrow geometrical dimensional range.

In our recent previous work, ${ }^{13}$ we demonstrated that for the case of a nanocantilever beam under static bending deformation [see Fig. 1(a)], the apparent piezoelectric coefficient is considerably increased over the corresponding bulk value. Both a theoretical model (supplemented with experimental material properties) and quantum mechanical based molecular dynamics simulations were employed. The major results are summarized in Fig. 1(b). Our results indicate a significant increase of $80 \%$ in the apparent piezoelectric response of paraelectric nonpiezoelectric (bottom curves) $\mathrm{BaTiO}_{3}$ (BT) (cubic phase) at beam thickness of $5 \mathrm{~nm}$. Moreover, in the case of piezoelectric (top curves) $\mathrm{BaTiO}_{3}$ (tetragonal phase), the apparent piezoelectric coefficient is enhanced by $500 \%$ over $\mathrm{BaTiO}_{3}$ bulk piezoelectric constant for the same beam thickness. We proved that the observations manifest in our atomistic calculations [summarized in Fig. 1(b)] are due to the flexoelectric effect. A nonuniform strain field or the presence of strain gradients can locally break inversion symmetry and induce polarization even in centrosymmetric crystals (as well evident in the case of cubic BT). This phenomenon is termed flexoelectricity. ${ }^{14,15}$ Phenomenologically we may write the polarization $P$ vector in terms of strain $S$ and its gradient as 


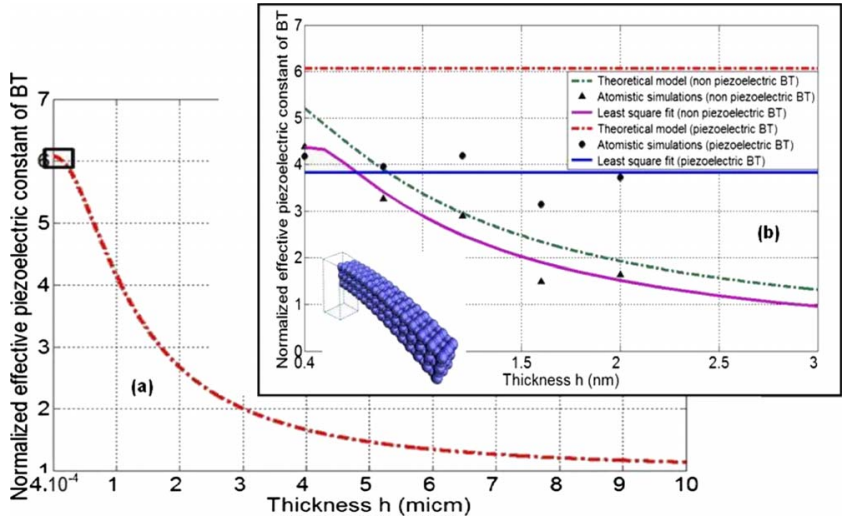

FIG. 1. (Color online) Normalized effective piezoelectric constant of BT. (a) Normalized effective piezoelectric constant of tetragonal (piezoelectric) BT (red dashed line). Enhancement starts at a higher length scale of a few micrometers and reaches significant magnitude at the nanosize. (b) The right inset corresponds to a zoomed-in view around $3 \mathrm{~nm}$ illustrating the summary of our results for both tetragonal (piezoelectric top curves) and cubic (nonpiezoelectric bottom curves) BT. The atomistic results show good agreement with the theoretical predictions.

$$
P_{i}=d_{i j k} S_{j k}+f_{i j k l} \nabla_{l} S_{j k}
$$

Here " $f$ " is the so-called fourth-order flexoelectric tensor. Thus, unlike the components of the third-order tensor " $d$ " (piezoelectric coefficients) that are nonzero for only selected (piezoelectric) dielectrics, the flexoelectric coefficients (components of the tensor " $f$ ") are, in principle, nonzero for all dielectrics (although, of course, they may be negligibly small for many materials). Recently, one of us ${ }^{16}$ has developed a complete mathematical framework for this phenomenon although various other works ${ }^{17-19}$ exist, which provide comparable information. Flexoelectricity is found to play an important role in the characteristics of ferroelectrics and has recently been a focus of several theoretical and experimental studies. $^{20-23}$ For example, Cross and co-workers ${ }^{24-30}$ well characterized the flexoelectricity of many ferroelectrics and suggested (along with others ${ }^{31}$ ) tantalizing applications. Besides the numerical value of the flexoelectric coefficients, exhibiting large values even for paraelectric phases of ferroelectrics, the strength of the flexoelectric size effect will depend also on how large the strain gradients are. The latter is closely linked with the size scale of the structure since strain gradients scale inversely with size (i.e., for the same strain in a cantilever beam, the strain gradients will be orders of magnitude larger at nanoscale as compared with micron scale).

Since the harvested power crucially depends upon the electromechanical coupling coefficient, we expect a considerable enhancement of the beam harvested power due to flexoelectricity at the nanoscale. This notion is the central premise of our present Rapid Communication.

Guided by the insights of our atomistic study on static piezoelectric beams, herein we analyze the response of resonant piezoelectric nanobeams under dynamical mechanical excitation for the purposes of energy harvesting. To highlight the physical insights for this Rapid Communication, we con- sider a simple paradigmatical model (which in fact is also quite the pragmatic choice in terms of applications). In the following, we consider a cantilever beam with crosssectional area $A$ ( $y-z$ plane) and length $l$ along the $x$ axis. The free end of the beam is subjected to external time-dependent mechanical loading mimicking ambient excitations.

The modified Hamilton's principle can be used to derive the governing differential equations,

$$
\int_{t_{1}}^{t_{2}} \delta(T-U) d t+\int_{t_{1}}^{t_{2}} \delta W d t=0,
$$

where $L=T-U$ is the Lagrangian and $W$ designates the work done by nonconservative forces.

The strain energy $U$ in the present context (i.e., including flexoelectric and polarization gradient coupling) can be defined as

$$
U=\frac{1}{2} \iint_{V} \int T_{i j} S_{i j} d V+\frac{1}{2} \iint_{V} \int \tilde{T}_{i j k} u_{i, j k} d V,
$$

where $u$ is the displacement vector, $S$ is the strain tensor, $T$ and $\tilde{T}$ are, respectively, the Cauchy stress and higher order stress tensors given by the following constitutive equations established previously:

$$
\begin{gathered}
T_{i j}=c_{i j k l} S_{k l}+e_{i j k l} P_{k, l}+d_{i j k} P_{k}, \\
\widetilde{T}_{i j k}=f_{i j k l} P_{l},
\end{gathered}
$$

where $P$ is the polarization vector, $c, e, d$, and $f$, respectively, designate elastic tensor, polarization gradient and strain coupling tensor introduced by Mindlin, ${ }^{17}$ piezoelectric tensor, and flexoelectric tensor. It is also worth to point out that the piezoelectric coefficients $d$ and the polarization $P$ can themselves be size dependent (beyond the mechanism of flexoelectricity) as has been demonstrated experimentally ${ }^{32}$ and theoretically $^{33}$ for ferroelectric nanowires. In order not to becloud the central point of this Rapid Communication, this aspect is not considered in what follows.

Here, we employ the typical simplifying assumptions attendant on a thin slender structure; i.e., the only nonzero strain components is the axial strain $S_{1}$ that can be explicitly written in terms of the beam deflection $w$ as

$$
S_{1}(x, z)=-z \frac{d^{2} w(x)}{d x^{2}} .
$$

Obviously, any deformation along the longitudinal direction also induces a deformation of the opposite sign in the transverse direction, although this effect is neglected in a beamlike analysis and is expected to be negligible in slender beams.

The kinetic energy is simply

$$
T=\frac{1}{2} \int_{V} \rho\left[\frac{\partial w(x, t)}{\partial t}\right]^{2} d v,
$$

where $\rho$ is the mass density.

There are two contributions to the work done by nonconservative forces: external forces [say, transversal load $q(x, t)]$ 
and frictional forces. The work done by nonconservative forces is then

$$
W=\int_{0}^{l} q(x, t) w(x, t) d x-\int_{0}^{l} r_{b} \frac{\partial w(x, t)}{\partial t} w(x, t) d x .
$$

We suppress further details (which will also be presented elsewhere). Essentially, variational minimization of Eq. (2) leads to the following final result for the dynamical bending response of a piezoelectric nanobeam in which flexoelectric size effects are incorporated:

$$
\mu \frac{\partial^{2} w(x, t)}{\partial t^{2}}+G \frac{\partial^{4} w(x, t)}{\partial x^{4}}+r_{b}\left[\frac{\partial w(x, t)}{\partial t}\right]=q(x, t),
$$

where $\mu$ and $r_{b}$ are, respectively, the mass and the friction coefficient per unit length of the beam. $G$ is the beam "renormalized" bending rigidity defined as

$$
\begin{aligned}
& G=[\underbrace{Y I}_{\text {pure elasticity }}+\underbrace{Y I \xi}_{\text {pure piezoelectricity }}+\underbrace{A f f^{\prime}}_{\text {pure flexoelectricity }} \\
& +\underbrace{\frac{A f \xi^{2}(e-f)}{d^{2} Y}}_{\text {piezoelectricity-flexoelectricity interaction }}] \text {. }
\end{aligned}
$$

$\xi=k_{e}^{2}=k^{2} /\left(1-k^{2}\right)$ is defined as the square of the expedient coupling coefficient $k_{e}$ and $k=\sqrt{Y d^{2} / \varepsilon}$ is the so-called electromechanical coupling (EMC) coefficient. $I$ is the moment of inertia. $Y, \varepsilon_{0}$, and $\chi$ are, respectively, the Young's modulus, permittivity of the vacuum, and dielectric susceptibility. Here $e=e_{13}, f=f_{13}, f^{\prime}=f_{55}$, and $d=-d_{31} / Y$.

Equation (8) differs from the classical one mainly in that elastic, dielectric, and piezoelectric properties are renormalized due to the flexoelectric terms. In Eq. (9), the first term is the classical elasticity beam bending rigidity and the second term represents an extra contribution due to piezoelectricity. The last two terms correspond to additional contributions due to the existence of flexoelectricity. In particular, the third term is due to a pure strain gradient or flexoelectric effect, whereas the last term corresponds to a nonlinear interaction between flexoelectricity and piezoelectricity.

We now turn our attention to the energy harvesting aspect. A typical piezoelectric energy harvester ${ }^{34}$ is composed of a piezoelectric vibrating element and an energy storage system such as a battery. The piezoelectric vibrator is a collection of a mass $M$, a spring with stiffness $K$, and a damper with damping coefficient $\eta$. The electromechanical coupling is of course renormalized due to flexoelectricity and an "effective" value, $\xi_{\text {eff }}$, and can be defined from Eq. (9) as

$$
\xi_{\text {eff }}=\xi+\frac{A f f^{\prime}}{Y I}+\frac{A f \xi^{2}(e-f)}{d^{2} Y^{2} I} .
$$

Since the governing equations are found to be identical to the classical case (except for the renormalized size-dependent material parameters), we can directly write the analytical expression for the normalized harvested power $\bar{P}[$ Eq. (11)] of a piezoelectric energy harvesting system already derived by Shu and $\mathrm{Lien}^{34}$ as

$$
\begin{aligned}
\bar{P}= & \frac{P}{\frac{F_{0}^{2}}{w_{n}}}=\frac{1}{\left(r \Omega+\frac{\pi}{2}\right)^{2}} \\
& \times \frac{\xi_{\mathrm{eff}} \Omega^{2} r}{\sqrt{\left[2 \zeta+\frac{2 \xi_{\mathrm{eff}} r}{(r \Omega+\pi / 2)^{2}}\right]^{2} \Omega^{2}+\left(1-\Omega^{2}+\frac{\Omega \xi_{\text {eff }} r}{r \Omega+\pi / 2}\right)^{2}}} .
\end{aligned}
$$

The different parameters are, respectively, defined as natural frequency, damping ratio, normalized frequency, and normalized resistance,

$$
w_{n}=\sqrt{\frac{K}{M}}, \quad \zeta=\frac{\eta}{2 \sqrt{K M}}, \quad \Omega=\frac{w}{w_{n}}, \quad r=C_{p} w_{n} R,
$$

with $C_{p}$ being the piezoelectric capacitance, $R$ the load resistance, and $w$ the excitation angular frequency.

According to Eq. (11) the harvested power is dependent on $\Omega, r, \zeta$, and $\xi_{\text {eff }}$. Hence, we will use the values of system parameters from Shu and Lien $^{34}$ for a piezoelectric PZT beam harvester to be able to draw a comparison. The damping ratio used is $3 \%$.

Under the assumption of large electromechanical coupling $x=\xi_{\mathrm{eff}} / \zeta \gg 1$, two resonance modes can be distinguished:

(1) Short circuit resonance $\left(w=w_{n}\right.$ and $\left.\Omega=1, r=0.062\right)$. At short circuit conditions Eq. (11) turns out to be

$$
\bar{P} \approx \frac{r x}{\zeta\left[\left(\frac{\pi}{2}+\frac{2 r x}{\pi}\right)^{2}+(r x)^{2}\right]} .
$$

(2) Open circuit resonance $\left(\Omega=\sqrt{1+\xi_{\text {eff }}}, r=17.3\right)$. In the case of an open circuit, Eq. (11) becomes

$$
\bar{P} \approx \frac{r x \Omega^{2}}{\zeta\left[4 \Omega^{2}\left(r \Omega+\frac{x}{\Omega}\right)^{2}+\left(\frac{\pi}{2} x\right)^{2}\right]} .
$$

The results of the harvested power in both resonance conditions are depicted in Fig. 2 and contrasted with the classical case where flexoelectricity is ignored. The solid flat lines designate the anticipated harvested power for classical piezoelectric beams. The dashed lines correspond to our predicted harvested power for nanobeams incorporating the flexoelectric effect. The optimal beam thickness for which we obtain a maximum power generated is around $21 \mathrm{~nm}$ for the short circuit resonance and $26 \mathrm{~nm}$ in the case of the open circuit. The peak power value at short circuit resonance reaches almost twice the power obtained for the classical piezoelectric beam. In the case of the open circuit, the maximum harvested power is increased by $30 \%$.

Our results (Fig. 2) clearly demonstrate a size-dependent effect of the harvested power due to flexoelectricity. The enhancement commences around a couple of hundred nanometers $(10 \%$ for short circuit and $7 \%$ for open circuit at a 


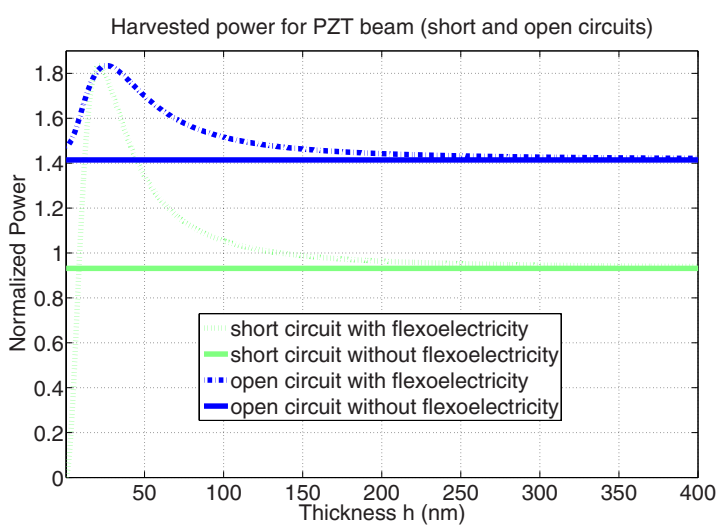

FIG. 2. (Color online) Harvested power as function of beam thickness for short and open circuit resonances. Solid lines correspond to the harvested power for classical piezoelectric beam. The dashed and dotted lines show a size dependency of the harvested power that nearly doubles for the short circuit (green or light gray) and is enhanced by $30 \%$ for the open circuit (blue or dark gray) when including flexoelectricity.

thickness of $100 \mathrm{~nm}$ ) and becomes significantly large reaching a maximum at optimal smaller sizes $(100 \%$ for short circuit at $21 \mathrm{~nm}$ and $30 \%$ for open circuit at $26 \mathrm{~nm}$ ). As the beam thickness increases, the flexoelectric effect becomes negligible and our simulated results match the classical piezoelectric case. Diminishing the size below a certain preferred size does not lead to any gains. When thickness decreases, the effective electromechanical coupling [defined in Eq. (10)] is enhanced. Hence, the asymptotic limits are $\lim _{x \rightarrow \infty} \bar{P} \approx \lim _{x \rightarrow \infty}(1 / x)=0$ for the short circuit resonance and $\lim _{x \rightarrow \infty} \bar{P} \approx c s t \neq 0$ for open circuit conditions according to Eqs. (13) and (14), respectively. The harvested power peak at small thicknesses is due to a resonance type of behavior. According to Eq. (9), flexoelectricity also renders the effective stiffness $K$ and hence the natural frequency $w_{n}$ size dependent. At fixed normalized resistance and damping ratio, as size varies, the effective electromechanical coupling and natural frequency of the system will change accordingly until a resonance occurs at a critical or resonant thickness characterized by a peak of the harvested power. Inspired from several works on flexoelectricity in which the noncentrosymmetry of the components shape is found to be important, ${ }^{31,35}$ we have also rederived our results for the peak power of a triangular shaped cantilever beam and find that the peak power can be doubled (200\% increase) when compared to a rectangular one.

In summary, we have shown that within a narrow range of geometrical parameters at the nanoscale, a dramatic increase in the energy harvesting capability is expected for piezoelectric nanostructures. This insight is expected to provide a scheme for engineering energy scavenging via piezoelectrics and our results provide guidelines for the experimental detection of the predicted effect for PZT. Our results are expected to be applicable to other piezoelectrics as well, especially those that exhibit a high flexoelectric constant such as $\mathrm{BaTiO}_{3}$.

P.S. and M.S.M. acknowledge financial support from the NSF under NIRT Grants No. CMMI 0708096 and No. CMMI 0826153.
*Corresponding author. psharma@uh.edu

${ }^{1}$ L. Mateu and F. Moll, J. Intell. Mater. Syst. Struct. 16, 835 (2005).

${ }^{2}$ N. S. Shenck and J. A. Paradiso, IEEE MICRO 21, 30 (2001).

${ }^{3}$ J. Granstrom et al., Smart Mater. Struct. 16, 1810 (2007).

${ }^{4}$ H. A. Sodano et al., Shock Vib. Dig. 36, 197 (2004).

${ }^{5}$ S. R. Anton and H. A. Sodano, Smart Mater. Struct. 16, R1 (2007).

${ }^{6}$ S. P. Beeby et al., Meas. Sci. Technol. 17, R175 (2006).

${ }^{7}$ S. Priya, J. Electroceram. 19, 165 (2007).

${ }^{8}$ S. Roundy et al., IEEE Pervasive Comput. 4, 28 (2005).

${ }^{9}$ Z. L. Wang and J. Song, Science 312, 242 (2006).

${ }^{10}$ J. Song et al., Nano Lett. 6, 1656 (2006).

${ }^{11}$ X. D. Wang et al., Science 316, 102 (2007).

${ }^{12}$ Z. Wang et al., Nano Lett. 7, 2966 (2007).

${ }^{13}$ M. S. Majdoub et al., Phys. Rev. B 77, 125424 (2008).

${ }^{14}$ E. V. Bursian and N. N. Trunov, Fiz. Tverd. Tela (Leningrad) 16, 1187 (1974).

${ }^{15}$ G. Catalan et al., J. Phys.: Condens. Matter 16, 2253 (2004).

${ }^{16}$ R. Maranganti et al., Phys. Rev. B 74, 014110 (2006).

${ }^{17}$ R. D. Mindlin, Int. J. Solids Struct. 4, 637 (1968).
${ }^{18}$ J. P. Nowacki and R. K. T. Hsieh, Int. J. Eng. Sci. 24, 1655 (1986).

${ }^{19}$ E. Sahin and S. Dost, Int. J. Eng. Sci. 26, 1231 (1988).

${ }^{20}$ T. Dumitrica et al., Chem. Phys. Lett. 360, 182 (2002).

${ }^{21}$ S. V. Kalinin and V. Meunier, Phys. Rev. B 77, 033403 (2008).

${ }^{22}$ P. Zubko et al., Phys. Rev. Lett. 99, 167601 (2007).

${ }^{23}$ P. Zubko et al., Phys. Rev. Lett. 100, 199906(E) (2008).

${ }^{24}$ J. Fousek et al., Mater. Lett. 39, 287 (1999).

${ }^{25}$ W. Ma and L. E. Cross, Appl. Phys. Lett. 79, 4420 (2001).

${ }^{26}$ W. Ma and L. E. Cross, Appl. Phys. Lett. 81, 3440 (2002).

${ }^{27}$ W. Ma and L. E. Cross, Appl. Phys. Lett. 82, 3923 (2003).

${ }^{28}$ L. E. Cross, J. Mater. Sci. 41, 53 (2006).

${ }^{29}$ J. Y. Fu et al., J. Appl. Phys. 100, 024112 (2006).

${ }^{30}$ M. Wenhui and L. E. Cross, Appl. Phys. Lett. 88, 232902-1 (2006).

${ }^{31}$ N. D. Sharma et al., J. Mech. Phys. Solids 55, 2328 (2007).

${ }^{32}$ J. E. Spanier et al., Nano Lett. 6, 735 (2006).

${ }^{33}$ H. Fu and L. Bellaiche, Phys. Rev. Lett. 91, 257601 (2003).

${ }^{34}$ Y. C. Shu and I. C. Lien, Smart Mater. Struct. 15, 1449 (2006).

${ }^{35}$ W. Zhu et al., Appl. Phys. Lett. 89, 192904-1 (2006). 